\title{
Using etoposide + dexamethasone-based regimens to treat nasal type extranodal natural killer/T-cell lymphoma-associated hemophagocytic lymphohistiocytosis
}

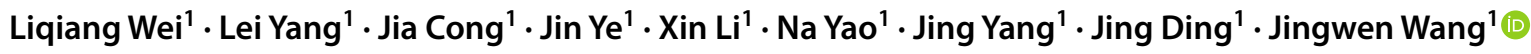

Received: 29 May 2020 / Accepted: 24 August 2020 / Published online: 6 October 2020

(c) Springer-Verlag GmbH Germany, part of Springer Nature 2020

\begin{abstract}
Purpose Nasal type extranodal natural killer/T-cell lymphoma (ENKTL) can be associated with hemophagocytic lymphohistiocytosis (NK/T-LAHLH), which is a rare and fatal disease with no effective therapy. We evaluated whether etoposide + dexamethasone-based chemotherapy regimens might be useful for treating NK/T-LAHLH.

Methods This retrospective single-center study evaluated clinical data from 37 patients with NK/T-LAHLH who were treated between May 2008 and January 2020.

Results Among 363 patients with ENKTL, the cumulative incidence of HLH was 11.9\%. Among 43 patients with NK/TLAHLH, 37 patients received etoposide + dexamethasone-based chemotherapy regimens, with an overall response rate of 45.9\% for the HLH. The overall response rate was substantially higher for newly diagnosed NK/T-LAHLH than it was for relapsed or refractory NK/T-LAHLH (66.7\% vs. $18.8 \%)$. The median overall follow-up time was 4 months, with overall survival rates of $81.1 \%$ at 1 month, $62.2 \%$ at 2 months, $56.8 \%$ at 3 months, and $34.4 \%$ at 6 months. Significantly better overall survival (all $P<0.05$ ) was observed for patients with newly diagnosed NK/T-LAHLH (vs. relapsed/refractory disease), stage I/II disease (vs. stage III/IV disease), and nasal disease (vs. non-nasal disease). Patients who responded to the ENKTL treatment also experienced response in their HLH; 8 patients experienced continued complete response for both HLH and ENKTL. Multivariate analysis revealed that a poor prognosis among patients with NK/T-LAHLH was independently related to relapsed/refractory ENKTL and non-nasal disease.

Conclusion Although patients with NK/T-LAHLH generally experienced poor outcomes, etoposide + dexamethasone-based chemotherapy regimens were associated with good outcomes among select patients with newly diagnosed or stage I/II NK/T-LAHLH.
\end{abstract}

Keywords Extranodal NK/T-cell lymphoma, nasal type $\cdot$ Hemophagocytic lymphohistiocytosis $\cdot$ Chemotherapy Etoposide $\cdot$ Dexamethasone

\section{Introduction}

Nasal type extranodal NK/T-cell lymphoma (ENKTL) is a relatively rare invasive non-Hodgkin lymphoma (NHL) that is closely associated with the Epstein-Barr virus (Swerdlow et al. 2017). In China, ENKTL is the most common NK/T-cell lymphoma, where it accounts for $12.0-17.1 \%$ of NHL cases

Jingwen Wang

wjwtrxy@126.com

1 Department of Hematology, Beijing Tongren Hospital, Capital Medical University, No. 1 Dong Jiao Min Xiang Street, Dong Cheng District, Beijing 100730, China
(Yang et al. 2011; Sun et al. 2012). Recent research has helped improve treatment outcomes, although patients with advanced stage or relapsed/refractory ENKTL (R/R-ENKTL) still have a very poor prognosis, with an overall response rate (ORR) of $60-70 \%$, a complete response (CR) rate of $40-50 \%$, and a 5-year overall survival (OS) rate of 30-50\% (Yamaguchi et al. 2018). Patients with advanced disease, a high tumor burden, and R/R-ENKTL often experience hemophagocytic lymphohistiocytosis (HLH), which has an extremely high mortality rate and has become an important cause of death among patients with ENKTL (Liu et al. 2019; Emile et al. 2016).

HLH involves an excessive inflammatory response that is mainly caused by abnormal activation and proliferation of lymphocytes, monocytes, and macrophages, which lead to 
the secretion of a large number of inflammatory cytokines. The clinical manifestations are persistent fever, hepatosplenomegaly, and multiple organ failure, while the laboratory test abnormalities include cytopenia, hyperferritinemia, abnormal triglyceride and fibrinogen concentrations, and hemophagocytosis in the bone marrow, liver, spleen, and lymph nodes (Malinowska et al. 2014). The reported mortality rate of HLH is $20-88 \%$ (Hayden et al. 2016). Cases involving HLH can be classified as primary or secondary, with primary HLH typically caused by chromosomal or genetic abnormalities, while secondary HLH is often triggered by serious underlying diseases. Secondary HLH is commonly associated with infectious diseases and neoplasms. It was reported that nearly a third of reported cases in adults had more than one underlying cause. T-cell or natural-killer lymphoma (16.8\%), B-cell lymphoma (15.2\%), Epstein-Barr virus (15.0\%), HIV (7.9\%), systemic lupus erythematosus $(6.1 \%)$, and mycobacoterium tuberculosis (3.6\%) accounted for the largest proportion of triggers and associated diseases and processes reported in 2197 adult hemophagocytic lymphohistiocytosis cases (Ramos-Casals et al. 2014). Two studies have indicated that $7.1-11.4 \%$ of ENKTL cases are complicated with HLH, and these patients have extremely poor outcomes with a median survival time of approximately 30 days (Li et al. 2017; Jia et al. 2016).

Most HLH treatments are based on the HLH-2004 strategy, although their efficacies vary greatly and the prognosis of HLH patients remains very poor (Hayden et al. 2016; Henter et al. 2007; Bubik et al. 2020; La Rosée et al. 2019). Some researchers have evaluated monoclonal antibodies targeting CD52 and TNF- $\alpha$ for treating HLH (Bubik et al. 2020; Keith et al. 2012), while other studies have confirmed that etoposide and dexamethasone (two key agents in the HLH-2004 strategy) play important roles in the treatment of HLH (Bergsten et al. 2017; Johnson et al. 2014). Therefore, for the past 12 years, we have used etoposide + dexamethasone-based chemotherapy regimens to treat NK/T-LAHLH. This retrospective study evaluates our experience and the patients' outcomes.

\section{Patients and methods}

\section{Patients and inclusion criteria}

Between May 2008 and January 2020, we identified 43 patients who were diagnosed with ENKTL in our department and concurrently had or subsequently developed HLH. The inclusion criteria were: (i) a pathological diagnosis of ENKTL, (ii) fulfilled the criteria for HLH based on the judgment of three physicians and the HLH-2004 diagnosis and treatment guidelines, and (iii) received etoposide + dexamethasone-based chemotherapy regimens for $\geq 2$ weeks. The exclusion criteria were: (i) incomplete clinical and follow-up data and (ii) patients who refused further treatment. Based on these criteria, we included 37 of the 43 patients with NK/T-LAHLH.

The diagnosis of HLH was based on the HLH-2004 diagnosis and treatment guidelines (Henter et al. 2007), which require the patient to fulfill $\geq 5$ of the following requirements: (i) fever, (ii) splenomegaly, (iii) decreases in two or three blood parameters (hemoglobin: $<90 \mathrm{~g} / \mathrm{L}$, platelets: $<100 \times 10^{9} / \mathrm{L}$, neutrophils: $<1.0 \times 10^{9} / \mathrm{L}$ ), (iv) hypertriglyceridemia and/or hypofibrinogenemia (fasting triglycerides: $\geq 3.0 \mathrm{mmol} / \mathrm{L}$, fibrinogen: $\leq 1.5 \mathrm{~g} / \mathrm{L}$ ), (v) blood cells detected in the bone marrow, spleen, or lymph nodes without evidence of malignant disease, (vi) NK cell activity reduced or lacking based on the local laboratory indicators, (vii) ferritin of $\geq 500 \mu \mathrm{g} / \mathrm{L}$, and (viii) soluble CD25 (sIL-2R) of $\geq 2400 \mathrm{U} / \mathrm{mL}$.

\section{Chemotherapy regimens}

The patients started etoposide + dexamethasone-based chemotherapy regimens immediately after the diagnosis of NK/TLAHLH. The etoposide dose was $75 \mathrm{mg} / \mathrm{m}^{2}$ intravenously on days 1-3 and the dexamethasone dose was $20 \mathrm{mg} /$ day intravenously on days $1-4$. The other drugs contained in the etoposide + dexamethasone-based specific regimens are listed in Table 1.

\section{Response assessment}

The response of HLH to treatment was evaluated every 2 weeks based on previous reports (Marsh et al. 2013; Wang et al. 2015). The responses were categorized as CR, partial response (PR), or non-response, and the ORR was calculated as $\mathrm{CR}+\mathrm{PR}$. The main response indicators were ferritin, blood cell counts, triacylglycerol, blood phagocytosis, consciousness level, and sCD25 level. We defined CR as all of the above indicators returning to within their normal range. We defined PR as $\geq 2$ symptoms/laboratory indicators improving by $>25 \%$ using the following parameters: (i) sCD25 level decreased by $>33 \%$, (ii) ferritin and triacylglycerol decreased by $>25 \%$, (iii) neutrophil increases in the absence of blood transfusion (by $100 \%$ to $>0.5 \times 10^{9} / \mathrm{L}$ for patients with $<0.5 \times 10^{9} / \mathrm{L}$ or by $100 \%$ and normalization for patients with $0.5-2.0 \times 10^{9} / \mathrm{L}$ ), (iv) a decrease of $>50 \%$ for patients with alanine aminotransferase levels of $>400 \mathrm{U} / \mathrm{L}$. For patients who survived for $\geq 4$ months and received $\geq 3$ chemotherapy cycles, the ENKTL response was categorized as $\mathrm{CR}, \mathrm{PR}$, or stable/progressive disease according to the Lugano criteria (Cheson et al. 2014).

\section{Follow-up}

Patients who achieved CR or PR continued to receive their assigned chemotherapy regimen. Patients who did not 
Table 1 Etoposide + dexamethasone-based regimens for treating NK/T-LAHLH

\begin{tabular}{lr}
\hline & $n(\%)$ \\
\hline Etoposide + dexamethasone & $4(10.8)$ \\
LVP + VP16 & $16(43.3)$ \\
(L-Asparaginase or pegaspargase, vindesine, dexamethasone, and etoposide) & \\
SVILE & $4(10.8)$ \\
(Pegaspargase, ifosfamide, vindesine, dexamethasone, and etoposide) & $7(18.9)$ \\
$\begin{array}{l}\text { PD-GemOx + VP16 } \\
\text { (Pegaspargase, gemcitabine, oxaliplatin, dexamethasone, and etoposide) }\end{array}$ & \\
Personalized treatment with the above regimens & $6(16.2)$ \\
\hline
\end{tabular}

NK/T-LAHLH nasal type extranodal natural killer/T-cell lymphoma-associated hemophagocytic lymphohistiocytosis respond to the initial chemotherapy subsequently received rescue or palliative treatment. The OS interval was evaluated for all patients, which we defined as the time from the start of treatment for NK/T-LAHLH to the first instance of death or loss to follow-up.

\section{Statistical analysis}

All analyses were performed using SPSS software (version 16.0; SPSS Inc., Chicago, IL). Non-normally distributed variables were expressed as median (range). Survival curves were created using the Kaplan-Meier method and compared using the log-rank test. Multivariate analysis was performed using the Cox regression method to evaluate potential prognostic factors.

\section{Results}

\section{Patients and treatments}

The 37 patients' characteristics at their diagnosis of NK/TLAHLH are listed in Table 2. All 37 patients received etoposide + dexamethasone-based chemotherapy regimens, which were selected based on the patient's age, major organ functions, and previously used regimens for relapsed/refractory patients (Table 1). The median number of treatment cycles was 2 (range: 1-6). During the treatments, symptomatic supportive therapies were administered as necessary, such as intravenous immunoglobulin, condensed human albumin infusion, liver-protecting agents, blood transfusions, antibiotics, antiemetic agents, and nutritional support. In $21 \mathrm{HLH}$ patients with newly diagnosed ENKTL, 6 of them received radiotherapy. Seven of 16 HLH patients with relapsed/ refractory ENKTL received radiotherapy during the previous induction therapy. Usually, the radiotherapy was followed by chemotherapy when the diseases were localized. The dose of radiotherapy was 50-55 Gy.
Table 2 Patient characteristics at the HLH diagnosis

\begin{tabular}{lc}
\hline & $n(\%)$ \\
\hline Male sex & $27(73.0)$ \\
Age in years, median (range) & $39(15-67)$ \\
Ann Arbor Stage & \\
Stage I/II & $5(13.5)$ \\
Stage III/IV & $32(86.5)$ \\
Disease at HLH onset & \\
Newly diagnosed ENKTL & $21(56.8)$ \\
Relapsed/refractory ENKTL & $16(43.2)$ \\
Primary lesion location & \\
Nasal & $32(86.5)$ \\
Non-nasal & $5(13.5)$ \\
PINK grade & \\
Low & $2(5.4)$ \\
Intermediate & $10(27.0)$ \\
High & $25(67.6)$ \\
Elevated serum LDH & $34(91.9)$ \\
ECOG performance score & \\
$0-2$ & $4(10.8)$ \\
$3-4$ & $33(89.2)$ \\
\hline
\end{tabular}

$H L H$ hemophagocytic lymphohistiocytosis, ENKTL extranodal natural killer/T-cell lymphoma, nasal type, PINK prognostic index for NK/T-cell lymphoma, $L D H$ lactate dehydrogenase, ECOG Eastern Cooperative Oncology Group

\section{Short-term efficacy}

The HLH responses were evaluated every 2 weeks during the chemotherapy for all 37 patients, which revealed an ORR of $45.9 \%$ (CR: $21.6 \%$, PR 24.3\%). The ORR, CR, and $P R$ rates for the various patient subgroups are shown in Table 3. Among 15 patients who survived for $\geq 4$ months and received $\geq 3$ chemotherapy cycles (CR or PR for HLH), the CR rate was $40.0 \%$, the PR rate was $33.3 \%$, and the stable/ progressive disease rate was $26.7 \%$. All 15 patients achieved CR or PR for their HLH. 


\section{Long-term efficacy}

The median follow-up time for all 37 patients was 4 months (range: 1-67 months) and the median survival time was 4 months (95\% CI: 2.82-5.18 months). Significantly better median OS was observed among patients with newly diagnosed (ND)-NK/T-LAHLH than among patients with R/R-NK/T-LAHLH (not reached vs. 2 months [95\% CI: 1.35-2.65 months], $P=0.001$ ) (Fig. 1a). Significantly better median OS was also observed among patients with stage I/II disease than among patients with stage III/IV disease (not reached vs. 4 months [95\% CI: 2.47-5.53 months], $P=0.028$ ) (Fig. 1b). Furthermore, significantly better median OS was observed among patients with nasal disease than among patients with non-nasal disease (4 months [95\% CI: 2.18-5.82 months] vs. 1 month [95\% CI: 1.00-1.00 months], $P=0.008$ ) (Fig. 1c). Finally, patients who achieved CR had significant better OS than patients who did not achieve CR $(P=0.000)$ (Fig. 1d).

\section{Multivariate analyses of prognostic factors}

The multivariate Cox regression model was used to evaluate ENKTL stage, newly diagnosed or relapsed/refractory disease status, nasal or non-nasal disease location, Eastern Cooperative Oncology Group (ECOG) performance status, and serum lactate dehydrogenase concentration. The results revealed that poor survival outcomes were independently associated with relapsed/refractory disease and non-nasal disease among patients with NK/T-LAHLH (Table 4).

Table 3 The HLH responses in the different groups

\begin{tabular}{lrrrr}
\hline & \multicolumn{2}{l}{ Response (\%) } & $P$ value \\
\cline { 2 - 3 } & CR & PR & ORR & \\
\hline Disease at HLH diagnosis & & & & \\
ND-ENKTL $(n=21)$ & 38.1 & 28.6 & 66.7 & 0.004 \\
R/R-ENKTL $(n=16)$ & 0.0 & 18.8 & 18.8 & \\
Stage of ENKTL & & & & \\
I/II $(n=5)$ & 80.0 & 0.0 & 80.0 & 0.159 \\
III/IV $(n=32)$ & 12.5 & 28.1 & 40.6 & \\
Primary ENKTL location & & & & \\
Nasal $(n=32)$ & 25.0 & 25.0 & 50.0 & 0.348 \\
Non-nasal $(n=5)$ & 0.0 & 20.0 & 20.0 & \\
\hline
\end{tabular}

$C R$ complete response, $P R$ partial response, $O R R$ overall response rate, $H L H$ hemophagocytic lymphohistiocytosis, $N D$-ENKTL newly diagnosed extranodal natural killer/T-cell lymphoma, nasal type, $R / R$ ENKTL relapsed or refractory extranodal natural killer/T-cell lymphoma, nasal type

\section{Discussion}

The rapid progression of the severe inflammatory reaction in HLH is associated with a high mortality rate. Primary HLH is often caused by genetic abnormalities, which generally occur in children and adolescents. Secondary HLH does not involve any known genetic abnormalities and often develops with or after the progression of underlying diseases, such as malignant tumors, serious infections, and autoimmune diseases. In secondary HLH cases, the most common type of malignant tumor is NK/T-cell lymphoma and the most common infectious disease is EBV infection (Parikh et al. 2014). As ENKTL is a common NK/T-cell lymphoma and is closely related to EBV infection, it has become one of the most common underlying diseases for secondary HLH.

Development of HLH can occur at the initial ENKTL diagnosis or when the underlying disease progresses (Riviere et al. 2014; Li et al. 2014; Ramos-Casals et al. 2014). In our clinical practice, some patients present with HLH when they are diagnosed with advanced ENKTL, and almost all patients who have relapsed/refractory or end-stage ENKTL will also have HLH. The present study involved 21 cases of ND-NK/T-LAHLH and 16 cases of R/R-NK/T-LAHLH, and we omitted all patients who did not receive etoposide + dexamethasone-based chemotherapy regimens. Given that the included patients had clear ENKTL, the cases were judged to involve secondary HLH and we did not test for primary HLH-related genes.

The treatment of secondary HLH relies on blocking the mechanisms through which the underlying disease triggers HLH, as well as suppressing the over-active immune response. The current HLH treatments generally follow the HLH-2004 strategy, which is mainly based on etoposide, dexamethasone, and cyclosporin A. In this context, etoposide can inhibit topoisomerase II, which leads to doublestranded DNA breaks, and can inhibit the production of inflammatory cytokines, selectively deplete activated T-cells in the HLH mouse model, and improve survival (Johnson et al. 2014). Therefore, etoposide has been considered a key drug in the HLH-94 and HLH-2004 guidelines. Cases of lymphoma-related HLH require treatment of both the HLH and the lymphoma, and some experts have recommended etoposide-containing combination chemotherapy as soon as possible for these patients (Schram et al. 2015).

The present study evaluated etoposide + dexamethasonebased chemotherapy regimens for treating NK/T-LAHLH, as these drugs can theoretically control the progression of HLH. These regimens also included drugs that are commonly used to treat ENKTL, such as pegaspargase, ifosfamide, gemcitabine, oxaliplatin, and vindesine. The results revealed an ORR of $45.9 \%$ for HLH among the 37 patients with NK/TLAHLH, which is substantially higher than the previously 

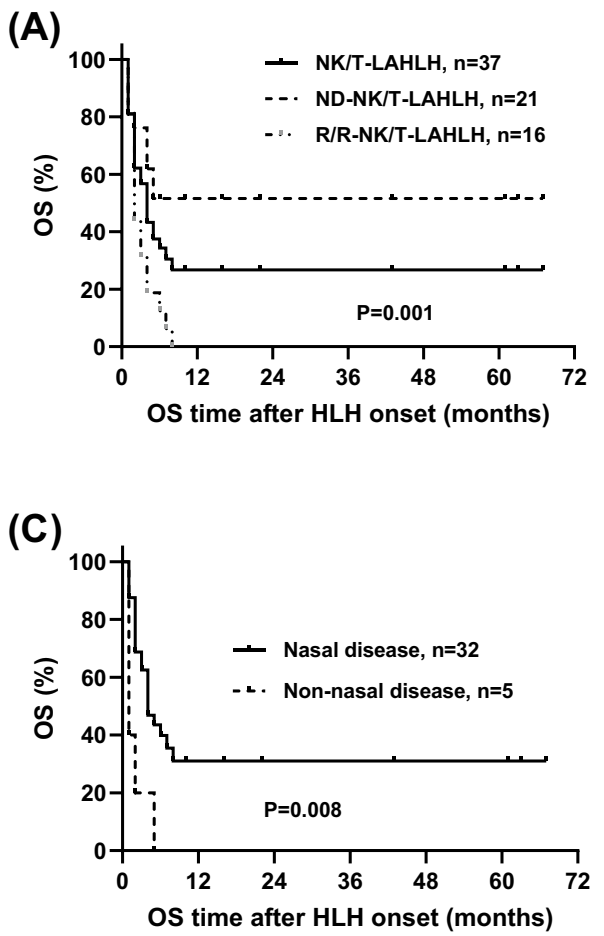

Fig. 1 Overall survival among the various groups of patients with natural killer/T-cell lymphoma-associated hemophagocytic lymphohistiocytosis (NK/T-LAHLH). a The 37 patients had overall survival (OS) rates of $81.1 \%$ at 1 month, $62.2 \%$ at 2 months, $56.8 \%$ at 3 months, and $34.4 \%$ at 6 months. The patients with newly diagnosed NK/T-LAHLH (ND-NK/T-LAHLH) had OS rates of $81.0 \%$ at 1 month, $76.2 \%$ at 2 months, $76.2 \%$ at 3 months, and $51.6 \%$ at $\geq 6$ months. Patients with relapsed or refractory NK/T-LAHLH (R/R-NK/T-LAHLH) had OS rates of $81.2 \%$ at 1 month, $43.8 \%$ at 2 months, $31.2 \%$ at 3 months, $12.5 \%$ at 6 months, and $0 \%$ at 8 months. b Patients with stage I/II disease had an OS rate of $80.0 \%$

Table 4 Multivariate analysis of factors that were associated with OS

\begin{tabular}{llll}
\hline & HR & $95 \%$ CI & $P$-value \\
\hline R/R-NK/T-LAHLH & 3.292 & $1.356-7.995$ & 0.008 \\
Non-nasal disease & 3.380 & $1.186-9.638$ & 0.023 \\
Stage III/IV & 2.823 & $0.261-30.538$ & 0.393 \\
ECOG score 3-4 & 2.095 & $0.227-19.309$ & 0.514 \\
Elevated serum LDH & 1.908 & $0.352-10.338$ & 0.454 \\
\hline
\end{tabular}

$O S$ overall survival, $H L H$ hemophagocytic lymphohistiocytosis, $H R$ hazard ratio, $C I$ confidence interval, $R / R-N K / T-L A H L H$ relapsed or refractory nasal type extranodal natural killer/T-cell lymphoma-associated hemophagocytic lymphohistiocytosis, ECOG Eastern Cooperative Oncology Group, $L D H$ lactate dehydrogenase

reported rate of $17.4 \%$ (Jia et al. 2016). Further analysis revealed that the ORR was substantially higher among patients with ND-NK/T-LAHLH than among patients with R/R-NK/T-LAHLH. This may indicate that the lymphoma cells of patients with ND-ENKTL were more sensitive to chemotherapy drugs during the induction therapy. In the
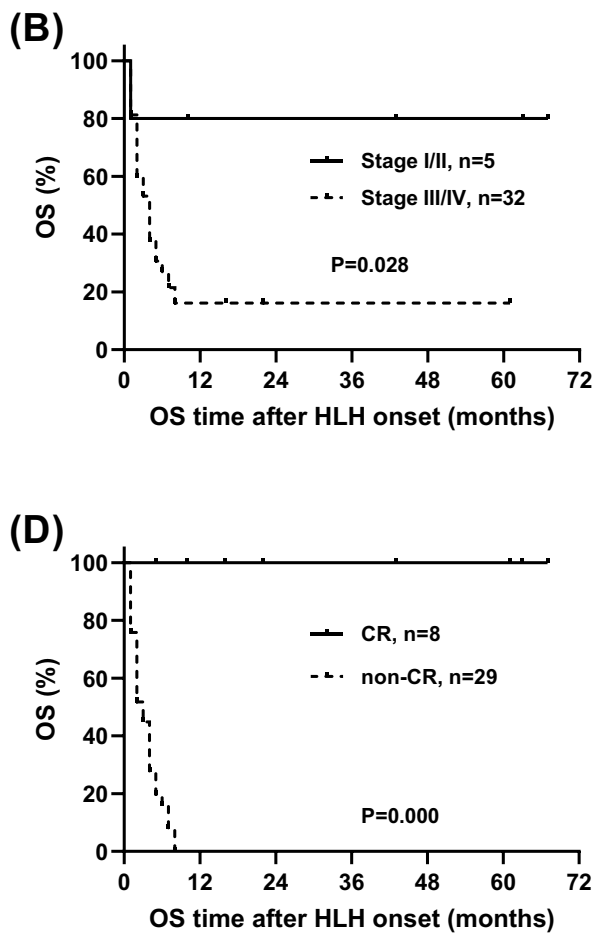

at $\geq 1$ month, while patients with stage III/IV disease had OS rates of $81.2 \%$ at 1 month, $59.4 \%$ at 2 months, $62.3 \%$ at 3 months, $39.9 \%$ at 6 months, and $16.1 \%$ at 8 months. c Patients with nasal disease had OS rates of $87.5 \%$ at 1 month, $68.8 \%$ at 2 months, $62.3 \%$ at 3 months, $39.9 \%$ at 6 months, and $31.0 \%$ at 8 months. Patients with non-nasal disease had OS rates of $40.0 \%$ at 1 month, $20.0 \%$ at 2 months, $20.0 \%$ at 3 months, and $0 \%$ at 5 months. d Patients who experienced complete response (CR) of their HLH had an OS rate of $100 \%$ during the follow-up period, although patients who did not experience CR had a median survival of only 2 months

ND-NK/T-LAHLH cases, the treatments appear to rapidly reduce the tumor burden, reduce or eliminate B symptoms, control the processes triggering HLH, and reduce the inflammatory response. However, the R/R-NK/T-LAHLH cases involved previous chemotherapy and it would be difficult to manage the secondary drug resistance, which would allow the ENKTL to continuously trigger HLH and lead to poor outcomes. Efficacy was not observed among patients with stage I/II disease or non-nasal disease, although this may have been related to the small sample sizes.

From the beginning of treatment, patients with NK/TLAHLH had a high early mortality rate and a median survival time of only 4 months, which reflects the rapid and dangerous nature of HLH progression. Previous reports have also described very poor survival among NK/TLAHLH patients, based on a median survival time of only 40 days, and OS rates of $26 \%$ at 6 months, $17 \%$ at 1 year, and $13 \%$ at 2 years (Bigenwald et al. 2018). T-cell lymphoma complicated with HLH also has OS rates of only $40 \%$ at 6 months, $25 \%$ at 1 year, and $15 \%$ at 2 years (Bubik et al. 
2020; Bigenwald et al. 2018). Another study of 22 patients with NK/T-LAHLH revealed a 2-year OS rate of 30\% (calculated from the diagnosis of ENKTL), with an ORR of $17.4 \%$, and a median survival time of only 26 days from the HLH diagnosis (Jia et al. 2016). Moreover, a study of $42 \mathrm{NK} / \mathrm{T}-\mathrm{LAHLH}$ patients revealed OS rates of $48.9 \%$ at 1 month, $36.7 \%$ at 2 months, $28.8 \%$ at 3 months, $23.0 \%$ at 6 months, and $15.4 \%$ at 1 year (Jin et al. 2018). Interestingly, in a study of 295 ENKTL patients, 21 patients developed HLH and the OS among all patients did not exceed 3 months after the HLH occurred, with a median survival of only 35 days ( $\mathrm{Li}$ et al. 2017). While those studies revealed very poor outcomes, they failed to identify prognostic factors or focus on specific treatment options. In contrast, the present study evaluated etoposide + dexamethasone-based treatment of NK/T-LAHLH, and revealed relative good HLH response and OS rates (versus the previous studies), although the patients' overall outcomes remained poor. Interestingly, further analysis revealed that patients with $\mathrm{CR}$ or PR for ENKTL also responded to their HLH treatment. Moreover, among the 8 patients with CR for HLH, the OS rate was $100 \%$ throughout the follow-up and their ENKTL remained in sustained CR. This suggests that etoposide + dexamethasone-based chemotherapy regimens may help control HLH and effectively treat ENKTL.

Patients with R/R-NK/T-LAHLH and stage III/IV disease had poor treatment response and poor OS, which may be related to drug resistance and large tumor burdens, as these factors could limit the ENKTL response and make it difficult to reverse HLH. Moreover, our study included five patients with non-nasal disease at their ENKTL diagnosis (i.e., very extensive lesions) and these patients had no response to treatment with an extremely short survival interval. Further multivariate analysis revealed that poor outcomes among patients with NK/T-LAHLH were independently associated with R/R-ENKTL and non-nasal disease, although poor outcomes were not associated with ECOG performance status, elevated lactate dehydrogenase levels, or stage III/IV disease. It is possible that the lack of associations for these factors were related to much smaller samples of patients with stage I/II disease, good ECOG performance status, and normal lactate dehydrogenase concentrations.

The HLH is a rare disease that can be complicated by ENKTL. Therefore, same attention should be paid to treating HLH and lymphoma. Although etoposide + dexamethasone-based chemotherapy had a good effect in some patients with an NK/T-LAHLH status in our study, more effective treatments should be investigated, such as pembrolizumab and nivolumab, which have been recommended for use in relapsed/refractory ENKTL patients. Ruxolitinib, has also been demonstrated to be effective in the treatment of severe inflammation caused by coronavirus disease 2019 (COVID19) (Yang Cao et al. 2020; La Rosée et al. 2020). In the future, we will try to add the above new drugs to the etoposide + dexamethasone-based combination chemotherapy regimen to treat this rare and life-threatening disease to improve the prognosis.

\section{Conclusion}

Patients with NK/T-LAHLH still have a very poor prognosis, although etoposide + dexamethasone-based chemotherapy regimens may help control the HLH and ENKTL in patients with stage I/II disease or ND-NK/T-LAHLH. Some of these patients even achieved good short-term and longterm outcomes, which support the potential utility of these treatments in this setting.

Acknowledgements The authors thank the patients and clinical staff at our centre for their assistance in this study, as well as Editage (www. editage.cn) for English language editing.

Funding This study was supported by grants from Beijing Municipal Science \& Technology Commission (Grant No. Z151100004015089).

Data availability The data that support the findings of this study are available from the corresponding author upon reasonable request.

\section{Compliance with ethical standards}

Conflict of interest The authors declare no potential conflicts of interest.

Ethical approval In our institute, any research study that prospectively assigns human participants or groups of humans to one or more healthrelated interventions to evaluate the effects on health outcomes must be registered at clinical trial registry and approved by the Ethic Committee. Our study is a retrospective study. It only collected clinical data of patients, did not interfere with patient's treatment, and did not pose any risks to the patients' physiology. We did our utmost to protect the patients' private information from disclosure. Our study is a retrospective study and follows the principles of the Declaration of Helsinki. The Ethics Committee considers that our retrospective study does not need ethical approval. If this situation does not meet the requirements of Journal of Cancer Research and Clinical Oncology, we will try our best to seek approval from the ethics committee.

\section{References}

Bergsten E, Horne A, Arico M et al (2017) Confirmed efficacy of etoposide and dexamethasone in HLH treatment: long-term results of the cooperative HLH-2004 study. Blood 130:2728-2738. https ://doi.org/10.1182/blood-2017-06-788349

Bigenwald C, Fardet L, Coppo P et al (2018) A comprehensive analysis of lymphoma-associated haemophagocytic syndrome in a large French multicentre cohort detects some clues to improve prognosis. Br J Haematol 183:68-75. https://doi.org/10.1111/bjh.15506

Bubik RJ, Barth DM, Hook C et al (2020) Clinical outcomes of adults with hemophagocytic lymphohistiocytosis treated with 
the HLH-04 protocol: a retrospective analysis. Leuk Lymphoma 11:1-9. https://doi.org/10.1080/10428194.2020.1737684

Cao Y, Wei J, Zou L et al (2020) Ruxolitinib in treatment of severe coronavirus disease 2019 (COVID-19): a multicenter, single-blind, randomized controlled trial. J Allergy Clin Immunol 146(1):137146.e3. https://doi.org/10.1016/j.jaci.2020.05.019

Chang Y, Cui M, Fu X et al (2018) Lymphoma associated hemophagocytic syndrome: a single-center retrospective study. Oncol Lett 16:1275-1284. https://doi.org/10.3892/ol.2018.8783

Cheson B, Fisher R, Barrington S et al (2014) Recommendations for initial evaluation, staging, and response assessment of Hodgkin and non-Hodgkin lymphoma: the Lugano classification. J Clin Oncol 32:3059-3068. https://doi.org/10.1200/JCO.2013.54.8800

Emile JF, Abla O, Fraitag S et al (2016) Revised classification of histiocytoses and neoplasms of the macrophage-dendritic cell lineages. Blood 127(22):2672-2681. https://doi.org/10.1182/blood -2016-01-690636

Hayden A, Park S, Giustini D, Lee AY, Chen LY (2016) Hemophagocytic syndromes (HPSs) including hemophagocytic lymphohistiocytosis (HLH) in adults: a systematic scoping review. Blood Rev 30:411-420. https://doi.org/10.1016/j.blre.2016.05.001

Henter JI, Horne A, Arico M et al (2007) HLH-2004: diagnostic and therapeutic guidelines for hemophagocytic lymphohistiocytosis. Pediatr Blood Cancer 48:124-131. https://doi.org/10.1002/ pbc. 21039

Henzan T, Nagafuji K, Tsukamoto H et al (2006) Success with infliximab in treating refractory hemophagocytic lymphohistiocytosis. Am J Hematol 81:59-61. https://doi.org/10.1002/ajh.20462

Jia J, Song Y, Lin N et al (2016) Clinical features and survival of extranodal natural killer/T cell lymphoma with and without hemophagocytic syndrome. Ann Hematol 95:2023-2031. https ://doi.org/10.1007/s00277-016-2805-9

Jin Z, Wang Y, Wang J et al (2018) Multivariate analysis of prognosis for patients with natural killer/T cell lymphoma-associated hemophagocytic lymphohistiocytosis. Hematology 23:228-234. https://doi.org/10.1080/10245332.2017.1385191

Johnson TS, Terrell CE, Millen SH, Katz JD, Hildeman DA, Jordan MB (2014) Etoposide selectively ablates activated T cells to control the immunoregulatory disorder hemophagocytic lymphohistiocytosis. J Immunol 192:84-91. https://doi.org/10.4049/jimmu nol.1302282

Keith MP, Pitchford C, Bernstein WB (2012) Treatment of hemophagocytic lymphohistiocytosis with alemtuzumab in systemic lupus erythematosus. J Clin Rheumatol 18:134-137. https://doi. org/10.1097/RHU.0b013e31824e8d9b

La Rosée P, Horne A, Hines M et al (2019) Recommendations for the management of hemophagocytic lymphohistiocytosis in adults. Blood 133(23):2465-2477. https://doi.org/10.1182/blood.20188 94618

La Rosée F, Bremer HC, Gehrke I et al (2020) The Janus kinase 1/2 inhibitor ruxolitinib in COVID-19 with severe systemic hyperinflammation. Leukemia 34(7):1805-1815. https://doi.org/10.1038/ s41375-020-0891-0

Li J, Wang Q, Zheng W et al (2014) Hemophagocytic lymphohistiocytosis: clinical analysis of 103 adult patients. Medicine (Baltimore) 93:100-105. https://doi.org/10.1097/MD.0000000000000022
Li N, Zhang L, Liu J et al (2017) A clinical study of 21 patients with hemophagocytic syndrome in 295 cases diagnosed with nasal type, extranodal nature killer/T cell lymphoma. Cancer Biol Ther 18:252-256. https://doi.org/10.1080/15384047.2017.1295176

Liu YZ, Bi LQ, Chang GL, Guo Y, Sun S (2019) Clinical characteristics of extranodal NK/T-cell lymphoma-associated hemophagocytic lymphohistiocytosis. Cancer Manag Res 11:997-1002. https ://doi.org/10.2147/CMAR.S183784

Malinowska I, Machaczka M, Popko K et al (2014) Hemophagocytic syndrome in children and adults. Arch Immunol Ther Exp 62:385-394. https://doi.org/10.1007/s00005-014-0274-1

Marsh RA, Allen CE, McClain KL et al (2013) Salvage therapy of refractory hemophagocytic lymphohistiocytosis with alemtuzumab. Pediatr Blood Cancer 60:101-109. https://doi. org/10.1002/pbc. 24188

Parikh SA, Kapoor P, Letendre L, Kumar S, Wolanskyj AP (2014) Prognostic factors and outcomes of adults with hemophagocytic lymphohistiocytosis. Mayo Clin Proc 89:484-492. https://doi. org/10.1016/j.mayocp.2013.12.012

Ramos-Casals M, Brito-Zerón P, López-Guillermo A, Khamashta MA, Bosch X (2014) Adult haemophagocytic syndrome. Lancet 383:1503-1516. https://doi.org/10.1016/S0140-6736(13)61048-X

Riviere S, Galicier L, Coppo P et al (2014) Reactive hemophagocytic syndrome in adults: a retrospective analysis of 162 patients. Am J Med 127:1118-1125. https://doi.org/10.1016/j.amjme d.2014.04.034

Schram AM, Berliner N (2015) How I treat hemophagocytic lymphohistiocytosis in the adult patient. Blood 125:2908-2914. https:// doi.org/10.1182/blood-2015-01-551622

Sun J, Yang Q, Lu Z et al (2012) Distribution of lymphoid neoplasms in China: analysis of 4,638 cases according to the World Health Organization classification. Am J Clin Pathol 138:429-434. https ://doi.org/10.1309/AJCP7YLTQPUSDQ5C

Swerdlow SH, Campo E, Harris NL et al (2017) WHO classification of tumours of haematopoietic and lymphoid tissues, revised, 4th edn. IARC, Lyon

Wang Y, Huang W, Hu L et al (2015) Multicenter study of combination DEP regimen as a salvage therapy for adult refractory hemophagocytic lymphohistiocytosis. Blood 126:2186-2192. https://doi.org/10.1182/blood-2015-05-644914

Yamaguchi M, Suzuki R, Oguchi M (2018) Advances in the treatment of extranodal NK/T-cell lymphoma, nasal type. Blood 131:25282540. https://doi.org/10.1182/blood-2017-12-791418

Yang QP, Zhang WY, Yu JB et al (2011) Subtype distribution of lymphomas in Southwest China: analysis of 6,382 cases using WHO classification in a single institution. Diagn Pathol 6:77. https://doi. org/10.1186/1746-1596-6-77

Publisher's Note Springer Nature remains neutral with regard to jurisdictional claims in published maps and institutional affiliations. 